\title{
Ion energy scaling under optimum conditions of laser plasma acceleration from solid density targets
}

\author{
A. V. Brantov, E. A. Govras, and V. Yu. Bychenkov \\ P. N. Lebedev Physics Institute, Russian Academy of Science, Leninskii Prospect 53, \\ Moscow 119991, Russia, and Center for Fundamental and Applied Research, \\ VNIIA, ROSATOM, Moscow 127055, Russia \\ W. Rozmus \\ Theoretical Physics Institute, University of Alberta, Edmonton T6G 2E1, Alberta, Canada
}

(Received 7 May 2014; published 2 February 2015)

\begin{abstract}
A new, maximum proton energy, $\varepsilon$, scaling law with the laser pulse energy, $E_{L}$, has been derived for solid density foils from the results of 3D particle-in-cell simulations. Utilizing numerical modeling, protons are accelerated during interactions of the femtosecond relativistic laser pulses with the plain semitransparent targets of optimum thickness. The scaling, $\varepsilon \sim E_{L}^{0.7}$, has been obtained for the wide range of laser energies, different spot sizes, and laser pulse durations. Our results show that the proper selection of foil target optimum thicknesses results in a very promising increase of the proton energy with the laser intensity even in the range of parameters below the radiation pressure (light sail) regime. The proposed analytical model is consistent with numerical simulations.
\end{abstract}

DOI: 10.1103/PhysRevSTAB.18.021301

PACS numbers: 41.75.Jv, 52.38.Kd, 52.27.Ny, 52.65.Rr

\section{INTRODUCTION}

Ion acceleration by intense ultrashort laser pulses has led to many original applications such as triggering of nuclear reactions [1,2]; research of warm dense matter [3]; laboratory astrophysics [4]; radiography [5-7]; fast ignition [8]; and hadron therapy [9]. Both experiments [10,11] and simulations [12] have demonstrated an increase of ion energy with a corresponding reduction of the target thickness. The high contrast pulses of modern lasers [10,11] have enabled effective acceleration of ions from ultrathin foils that are semitransparent to laser light. In this regime, where target thickness does not exceed laser skin depth, a high-intensity laser light pulse expels electrons from the irradiated area of the foil in a forward direction. This causes ion acceleration from the entire target volume through the mechanism combining elements of the target normal sheath acceleration (TNSA) mechanism $[13,14]$ and the Coulomb explosion [15,16], i.e., the directed Coulomb explosion (DCE) [17]. One more regime, so-called "break-out afterburner" (BOA) [18], is also possible but it is relevant to somewhat thicker targets and longer pulses which require laser hole boring during the pulse-foil interaction. According to Ref. [18], the BOA regime involves generation of a forward directed electron beam and development of relativistic Buneman instability in order to convert the

Published by the American Physical Society under the terms of the Creative Commons Attribution 3.0 License. Further distribution of this work must maintain attribution to the author(s) and the published article's title, journal citation, and DOI. electron energy into ion acceleration. BOA generates a quasimonoenergetic ion bunch during the early phase of acceleration. Here we will explore how to realize the most effective DCE mechanism.

It is known that target thickness should be properly matched to the laser intensity [19] in order to obtain maximum ion energy. Although the optimum target thickness can be estimated in the order of magnitude from 2D particle-in-cell (PIC) simulations [19], the 3D PIC simulations are needed to correctly quantify target thickness for different laser intensities. The optimum target thickness was found in Ref. [19], wherein the square root energy scaling for the proton energy with laser intensity (energy) was also inferred. The square root intensity scaling for the maximum proton energy is also predicted by the TNSA model, which is valid for thick targets [20]. The 3D PIC simulations to find optimum target thickness for the laser pulse energy must also examine the dependence on different spot sizes and pulse durations. Such optimization should include a systematic study of laser light absorption (i.e., laser to electron energy conversion efficiency) in semitransparent targets, a study that will form an important part of our paper. Our paper will quantify how all parameters of the pulse affect laser energy conversion to hot electrons and define the effectiveness of high-energy proton production. We will present the results of our 3D optimization study with PIC code MANDOR [21] for acceleration of protons from thin targets triggered by femtosecond laser pulses. The main outcome of this paper is the dependence of maximum proton energies on the laser intensities under conditions of volumetric heating of optimum thickness targets. 
We also propose a simple theoretical model that estimates maximum ion energy using the effective temperature of the laser heated electrons. Since the electric field that accelerates ions depends on the charge separation, the ratio of the Debye length of laser heated electrons $\lambda_{D}$ to the foil thickness $l$ is the main controlling parameter of the theory. So far, only two asymptotic cases have been studied in detail: quasineutral expansion $\lambda_{D_{e}} \ll l$ [22] and Coulomb explosion $\lambda_{D_{e}} \gg l$ [23]. None of these theories are fully applicable to thin semitransparent targets. We will show that our model, which is valid for arbitrary $\lambda_{D_{e}} / l$, qualitatively explains numerical simulations when the ponderomotive dependence [14] of the effective electron temperature on the absorbed laser energy is used.

\section{OPTIMIZATION OF PROTON ACCELERATION USING 3D PIC SIMULATIONS}

It has been already shown that thin foils with optimum thicknesses are well suited for proton acceleration. Making use of shorter laser pulses or tight laser focusing also results in some increase of proton energies [24]. Building on these results, we have performed $3 \mathrm{D}$ simulations of proton acceleration by ultrashort ( $\tau=30 \mathrm{fs}$ FWHM duration) tightly focused (FWHM of the laser spot $d=4 \lambda$ ) laser pulses with the PIC code MANDOR [21]. As a reference we set the laser wavelength at $\lambda=1 \mu \mathrm{m}$. Laser intensity is varied from $I=5 \times 10^{18}$ to $I=5 \times 10^{22} \mathrm{~W} / \mathrm{cm}^{2}$, which corresponds to laser pulse energies from 0.03 to $300 \mathrm{~J}$. To examine pulse duration $(\tau)$ and spot size $(d)$ effects on proton acceleration, three additional sets of laser pulse parameters with the same full laser energy have been used: increased laser spot size, $d=6 \lambda$, and decreased one, $d=2 \lambda$, for $\tau=30 \mathrm{fs}$ and for the increased pulse duration, $\tau=150 \mathrm{fs}$, for the focal spot size $d=4 \lambda$. A very tight laser pulse focusing at $2 \lambda$ has been implemented by using the parabolic mirror simulation technique [25]. For larger focal spots, Gaussian laser pulses have been used.

The laser pulse was focused on the front side of the thin $\mathrm{CH}_{2}$ plasma foils, which are composed of electrons, hydrogen ions, and fully ionized carbon ions $\left(\mathrm{C}^{6+}\right)$. The target has an electron density of $200 n_{c}$ (where $n_{c}=$ $m_{e} / 4 \pi e^{2} \omega^{2}$ is the plasma critical density), which corresponds to a solid mass density of $\mathrm{CH}_{2}\left(1.1 \mathrm{~g} / \mathrm{cm}^{3}\right)$. The target thickness $l$ has been varied from $3 \mathrm{~nm}$ to $1 \mu \mathrm{m}$.

We performed several runs with different target thicknesses that have been chosen according to the theoretically predicted optimum length, $\lambda a_{0} n_{c} /\left(n_{e} \pi\right)$ [26], where $a_{0}=0.85 \sqrt{I\left[\mathrm{~W} / \mathrm{cm}^{2}\right] \lambda[\mu \mathrm{m}]^{2} \times 10^{-18}}$. The results of the simulations for maximum proton energies are shown in the top panel of Fig. 1. They clearly confirm the existence of an optimum target thickness for which protons reach maximum energies. The optimum target thickness corresponding to the maximum of proton energies (Fig. 1, bottom panel) grows linearly with laser field amplitude,
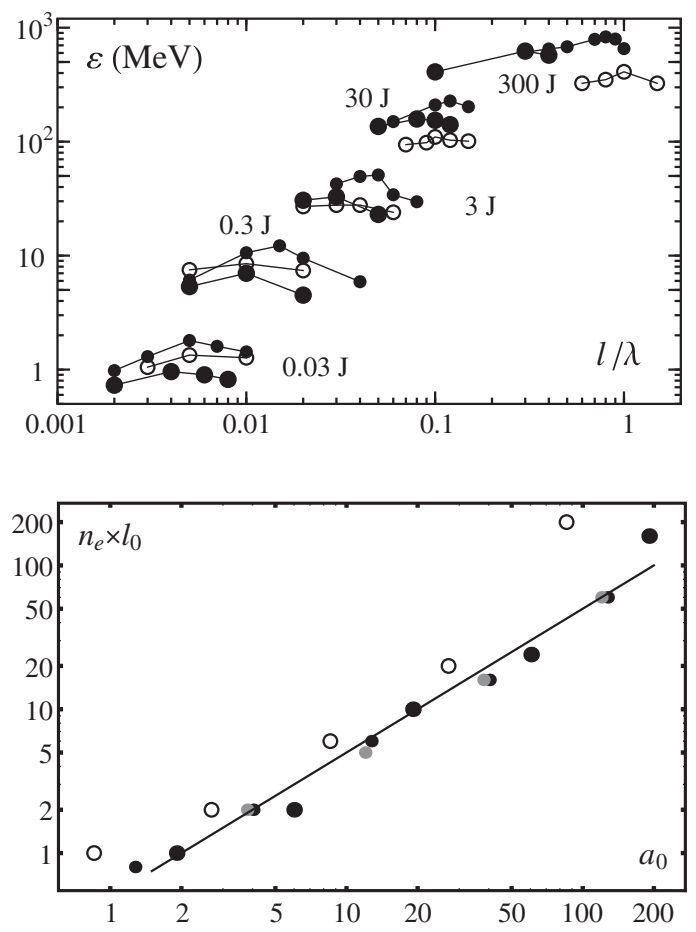

FIG. 1. Maximum proton energy vs target thickness for different laser energies (top panel) and optimum target areal density in $n_{c} \times \lambda$ units vs laser field $a_{0}$ (bottom panel). For both panels large black dots stand for $\tau=30 \mathrm{fs}$ and $d=4 \mu \mathrm{m}$, small black dots for $\tau=30 \mathrm{fs}$ and $d=6 \mu \mathrm{m}$, gray dots (only for the bottom panel because gray dots are indistinguishable from the large black dots in the upper panel) for $\tau=30 \mathrm{fs}$ and $d=2 \mu \mathrm{m}$, and open circles for $\tau=150$ fs and $d=4 \mu \mathrm{m}$.

$l_{0}=0.5 \lambda a_{0} n_{c} / n_{e}$, where the numeric factor 0.5 is almost independent from the focal spot size and it is slightly above the theoretically predicted $1 / \pi$ [26]. At the same time, this factor slightly increases with the laser pulse duration (see open circles in Fig. 1, bottom panel), i.e., the optimum regime of proton acceleration by the longer pulses requires thicker targets. The optimum target thickness corresponds to the partially transparent target [26], where volumetrically heated electrons are swept out of the plasma in a forward direction and give rise to strong charge separation fields [27], which accelerate protons by the DCE [17]. The laser pulse can then expel a large number of hot electrons from the focal spot region and penetrate inside the target in a hole-boring like action. This is the reason why optimal target thickness is somewhat above theoretically predicted values and this is why longer laser pulses can penetrate through thicker targets. Spectra of laser produced protons from the semitransparent volumetrically heated foils are similar to those resulted from the TNSA mechanism that are typical for thicker targets [28] (cf. Fig. 2). Our spectra do not demonstrate the BOA quasimonoenergetic feature.

Figure 3 shows the dependence of proton maximum energy on the laser pulse energy. The results of our simulations for the maximum energy of the protons from 


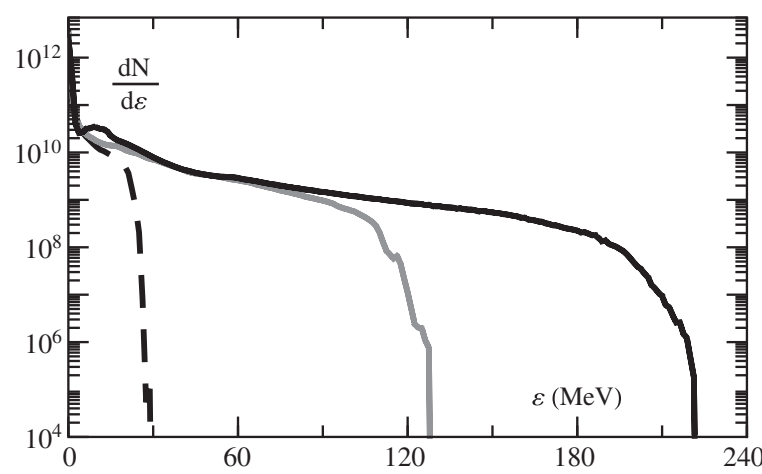

FIG. 2. The proton spectrum for optimum target thickness, $l_{0}=0.12 \lambda$, at time of $50 \mathrm{fs}$ (dashed line), $76 \mathrm{fs}$ (gray line) and $176 \mathrm{fs}$ (solid line) for laser with $E_{L}=30 \mathrm{~J}, \tau=30 \mathrm{fs}$, and $d=4 \mu \mathrm{m}$.

the optimum thickness targets are well approximated by the scaling $\varepsilon \propto E_{L}^{0.7}$, which is different from the square root dependence reported earlier [19]. The numerical proportionality factor varies from 14 for a long pulse $(150 \mathrm{fs})$ and large focal spot diameter $(d \gtrsim 4 \lambda)$, to 45 for tight laser focusing into the focal spot of $d=2 \lambda$. Given laser energies the shorter laser pulse and tighter focusing gives higher maximum proton energy. The number of energetic protons also increases with the laser energy. Note that long pulses are more effective for proton acceleration if the laser energy is less than $1 \mathrm{~J}$.

To better understand the proton energy scaling of Fig. 3, we have analyzed the laser energy absorption coefficient, $A$, for semitransparent targets. We define an absorption coefficient as the ratio of the particle kinetic energy to the initial laser energy. We found that for our parameters, the absorption coefficient of $30 \mathrm{fs}$ pulse in the targets with optimum thickness increases with laser energy (see Fig. 4) from values less than $10 \%$ for $0.03 \mathrm{~J}$ laser to $30 \%$ for $30 \mathrm{~J}$

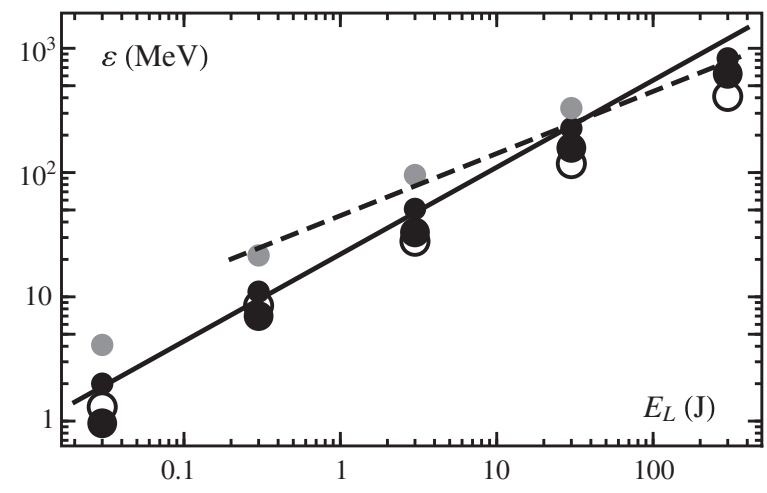

FIG. 3. Maximum proton energy for optimum target thickness vs laser energy for $\tau=30 \mathrm{fs}$ and $d=4 \mu \mathrm{m}$ (large black dots), $d=6 \mu \mathrm{m}$ (small black dots), $d=2 \mu \mathrm{m}$ (gray dots) and $\tau=$ $150 \mathrm{fs}$ and $d=4 \mu \mathrm{m}$ (open circles). The black and dashed lines correspond to the fits $\varepsilon[\mathrm{MeV}]=22\left(\mathrm{E}_{\mathrm{L}}[\mathrm{J}]\right)^{0.7}$ and $\varepsilon[\mathrm{MeV}]=$ $45\left(\mathrm{E}_{\mathrm{L}}[\mathrm{J}]\right)^{0.5}[19]$, correspondingly.

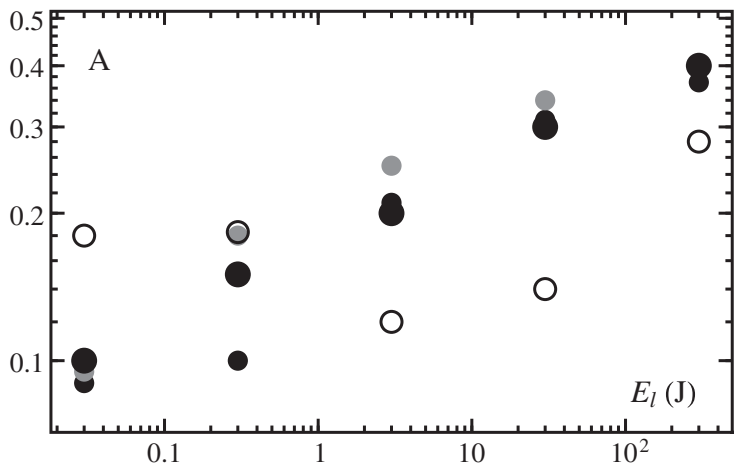

FIG. 4. Laser light absorption in the targets of optimum thickness as a function of the laser energy. Definitions of the dots are the same as in Fig. 3.

laser. The absorption of longer laser pulses is better for small laser energies. It is also not a monotonic function of the pulse energy. It drops from $18 \%$ to $12 \%$ at $3 \mathrm{~J}$ laser pulse energy and then grows to $\sim 30 \%$ for $300 \mathrm{~J}$ laser.

\section{THEORETICAL MODEL}

We will now demonstrate that the simple semianalytic theory qualitatively agrees with our simulation results and captures the main elements of interaction physics and ion acceleration. Assume that ion plasma occupies the layer of the width $l$ along the $x$ axis. The transversal size of the plasma is limited to the laser focal spot area, $\pi d^{2} / 4$. For simplicity, we consider one ion species plasma where ions are initially at rest within $-l / 2<x<l / 2$. During subsequent ion outflow, electrons remain in equilibrium with the electrostatic field and are described by the Boltzmann distribution function with an effective temperature $T$ : $n_{e}(x, t)=n_{e_{0}} \exp \left[e \varphi(x, t) / k_{B} T\right]$, where $n_{e_{0}}$ is initial electron density in the foil center. Electron temperature depends on laser intensity and can be time dependent due to adiabatic cooling after the pulse is terminated. Plasma expansion at $t>0$ will be symmetrical with respect to the plane $x=0$. For small distances $x_{f}<d$, where $x_{f}(t)$ is the ion front position, three-dimensional effects due to transversal size of the plasma region can be neglected and outflow is treated as one dimensional. Motion of ion plasma will be described by the following system of equations:

$$
\begin{aligned}
\epsilon_{0} \varphi^{\prime \prime} & =e n_{e}(x, t)-\operatorname{Zen}_{i}(x, t), \\
\left.\varphi^{\prime}\right|_{x=0} & =0,\left.\quad \varphi^{\prime}\right|_{x=\infty}=0, \\
M \ddot{x} & =-Z e \frac{\partial \varphi}{\partial x}, \quad \dot{x}(0)=0 \\
x(0) & =x_{0}, \quad 0 \leq x_{0} \leq l / 2, \\
n_{i}(x, t) & =n_{0}\left|\partial x / \partial x_{0}\right|^{-1} .
\end{aligned}
$$

From this point on we will use the following dimensionless variables: spatial coordinate $x$ is measured in the unit of 
$l / 2$, time $t$ is normalized to $1 / \omega_{p i}$ and densities $n_{i}, n_{e}$ to $n_{0}$, energies $Z k_{B} T$ and $Z e \varphi$ are written in terms of $(Z e)^{2} n_{0}(l / 2)^{2} / \epsilon_{0}$. The density $n_{e_{0}}$ is defined by initial ion density $n_{0}$, ion charge $Z$ and has a simple approximate dependence on the initial electron temperature $n_{e_{0}}=$ $Z n_{0}[1+2 T(0)]^{-1}$ [29]. Thus far there are no analytic solutions to Eq. (1) at arbitrary $T$. The only known solutions are for the case $\lambda_{D_{e}} \ll l$, i.e., for the nearly quasineutral expansion, $T \rightarrow 0$ [22] and for the case of $\lambda_{D_{e}} \gg l$, which is for the Coulomb explosion, $T \rightarrow \infty$ [23]. For the first case of the nearly quasineutral expansion $n_{i} \approx n_{e}$ and electric field at the ion front is $E_{1}=2 \sqrt{T} / \sqrt{2 e+t^{2}}$ [22], where $e=2.71828 \ldots$ In the second limit of a Coulomb explosion, one finds $n_{i}=1 / x_{f}(t)$ and $E_{1} \equiv 1$, where $x_{f}(t)$ is the front position of the expanding ion plasma [23].

To find an approximate solution of Eq. (1) for the arbitrary ratio of $\lambda_{D_{e}} / l$ and avoid complicated selfconsistent calculation of ion density we introduce the interpolating expression for $n_{i}(x, t)$ that is valid for an arbitrary temperature $T$. From the Poisson equation in Eq. (1) one obtains $E_{1}=\int_{0}^{x_{f}}\left[n_{i}(x, t)-n_{e}(x, t)\right] d x$. If we choose $n_{i}(x, t)=n_{e}(x, t)+E_{1} / x_{f}$ the expression for $E_{1}$ is satisfied and one obtains a correct asymptotics for both quasineutral outflow and for the Coulomb explosion regime. Solving the Poisson equation in (1) with such an ion density, we obtain an implicit expression for the function $E_{1}\left(x_{f}\right)$ :

$$
\left(E_{1}\right)^{-1}=1+\sqrt{\frac{x_{f} E_{1}}{2 T}} \exp \left[\frac{x_{f} E_{1}}{2 T}\right] \operatorname{Erf}\left[\sqrt{\frac{x_{f} E_{1}}{2 T}}\right] .
$$

Here $\operatorname{Erf}(z)=\int_{0}^{z} e^{-t^{2}} d t$. Note that Eq. (2) is simpler than that in Ref. [29] and has no interpolation coefficient.

A feature that may slightly affect density profile of the expanding plasma is the ion front singularity. It develops in our case due to decay of the initial sharp density profile with the discontinuity at $x=1$ ( $l / 2$ in dimensional units). These singularities, e.g., caustics, cusps, shocks, etc., have been discussed in many papers that are devoted to dynamics of noninteracting particles [30], dynamics of nondissipative gases in expanding universe [31], or Coulomb plasma expansion $[32,33]$. For the problem of a quasineutral plasma expansion this effect was first observed by Gurevich et al. in Ref. [34] and recently obtained by Allen et al. in Ref. [35]. Such fine density structures could not be resolved in our 3D PIC simulations. Careful examination of the spectra in Fig. 7 and especially the high energy front show no high energy spike in proton density that could result from the ion front singularity. However, we have performed 1D electrostatic BoltzmannVlasov-Poisson simulations (similar to those in Ref. [35]) with a sufficient number of particles and we have been able to resolve density peak near the ion front as shown in Ref. [35]. This is an integrable singularity that occurs in a very narrow spatial region and involves a very small number of particles. Also, this fine structure does not contribute noticeably to the electric field at the ion front and therefore has a negligible effect on the maximum ion energy. We also believe that in some cases these kinds of density singularities disappear if electron kinetic equation is used instead of the Boltzmann distribution. For example, in the previous paper [36] on the adiabatic plasma expansion that employed both electron and ion kinetic equations no density singularities were found for a sharp plasma boundary. On the other hand, for the initially smooth density profiles the singularities would appear.

We assume that plasma electrons are heated during the laser pulse duration $t<\tau$ and reach the characteristic temperature $T_{0}$. However, after that, $t>\tau$, they adiabatically cool down as described in [37]. Temporal behavior of electron temperature can be described as follows [37]:

$$
T(t)=T_{0}\left[\Theta(\tau-t)+\frac{\Theta(t-\tau)}{1+(t-\tau)^{2} / t_{c}^{2}}\right],
$$

where $\Theta(t)$ is the Heaviside step function and the characteristic cooling time is defined as $t_{c}=L / \sqrt{2} c_{s}$, where $L$ is the spatial scale of ion density and $c_{s}$ is the ion sound speed. We chose $L=x_{f}(\tau)$ and $c_{s}=\sqrt{T_{0}}$ as the typical parameters.

When ions reach the distance $x_{f} \sim L_{1}=1+d$ one must take into account a rapid decay of the accelerating field. Because of a finite volume occupied by ion charge in 3D, accelerating electric field decreases $\propto x^{-2}$ for $x_{f} \gg L_{1}$. Ensuring that the electric field satisfies two limits at the ion front, $E_{1}$ (2) for $x_{f}<L_{1}$ and $E_{1}\left(L_{1}\right) /\left(x-L_{1}\right)^{2}$ for $x_{f} \gg L_{1}$, one may propose a smooth connecting expression valid for the front position $x_{f}$ at the arbitrary distance from the foil. In addition, a laser pulse introduces asymmetry into plasma expansion because all electrons are accelerated by the laser pulse in a forward direction on the rear side of the thin foil. We will assume that the electric field at $x>0$ is twice the value $E_{1}$ (2) defined above for the symmetric expansion of the hot plasma layer into a vacuum. Consequently, the electric field at the position of the ion front at the arbitrary time can be approximately written as follows:

$$
E\left(x_{f}\right)= \begin{cases}2 E_{1}\left(x_{f}\right), & x_{f} \leq L_{1}, \\ 2 E_{1}\left(L_{1}\right)\left[1+\left(x_{f}-L_{1}\right)^{2}\right]^{-1}, & x_{f}>L_{1},\end{cases}
$$

where the time evolution of the electron temperature that contributes to $E_{1}$ (2) is given by Eq. (3). Solving the equation of motion (1) for the ions at the front of the expanding plasma with the electric field $E\left(x_{f}\right)$ (4) one obtains maximum ion energy $\varepsilon_{\max }=\left(\dot{x}_{f}\right)^{2} / 2$.

We show predictions of our theoretical model for the maximum ion energy in Fig. 5 for the hydrogen foil. The top panel displays dependence on the laser spot diameter, $d$, 

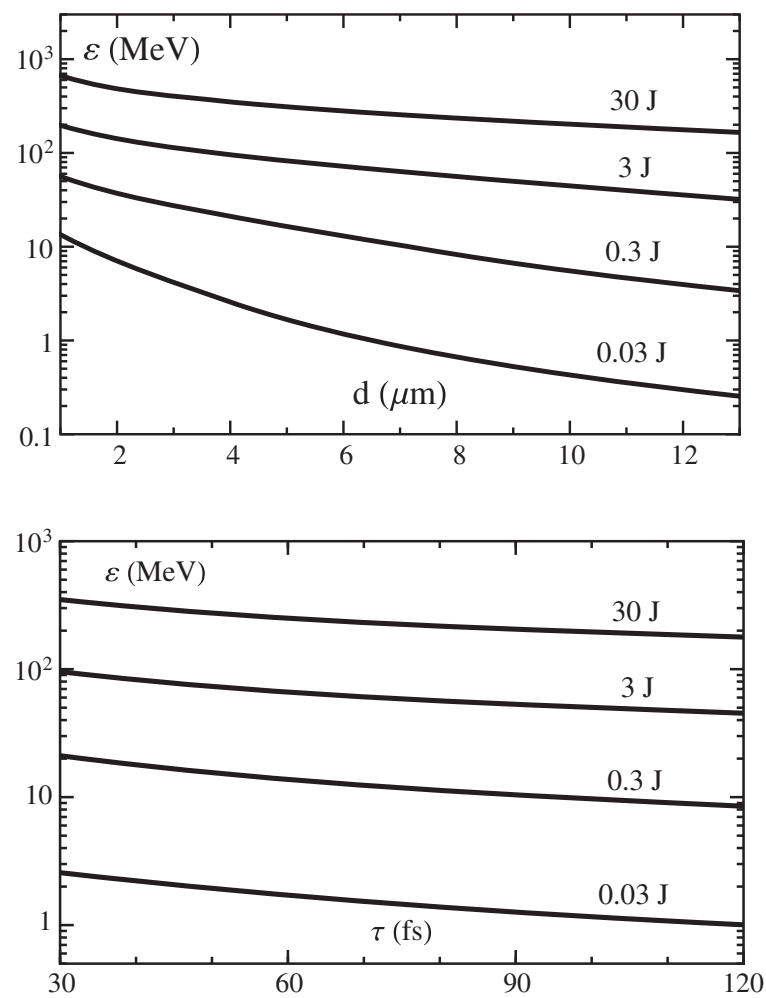

FIG. 5. Maximum proton energy as a function of the laser spot size $d$ (top panel) and as a function of the pulse duration (bottom panel) for a hydrogen foil of $n_{e}=200 n_{c r}$ and optimum thickness.

for the laser pulse duration of $\tau=30$ fs. The bottom panel shows maximum proton energy as the function of laser pulse duration for $d=4 \mu \mathrm{m}$. We have assumed that the electron temperature $T_{0}$ obeys the ponderomotive dependence [14], $T_{0} \propto m c^{2}\left[\sqrt{1+\left(a_{1}\right)^{2}}-1\right]$, where $a_{1}$ is the laser amplitude, calculated from the absorbed laser intensity $a_{1}=a_{0} \sqrt{A}$, and the foil is of the optimal thickness $l_{0}$. The curves in Fig. 5 agree with simulation results (cf. Fig. 3) which show that for a given pulse energy both laser light defocusing and pulse stretching cause the maximum ion energy to decrease.

In Fig. 6 we compare maximum energy of the protons for optimum target thicknesses from simulations (dots) and theory (lines).

It is clearly seen that the theory correctly reproduces the dependence of the maximum proton energy on laser intensity. Since our theory considers single-ion-species (H) foil, the curves for analytical solutions in Fig. 6 slightly overestimate simulation results (up to $30 \%$ for $\tau=30 \mathrm{fs}$ ). This is expected because carbon ions from $\mathrm{CH}_{2}$ foil are also accelerated thus making proton energy lower. Note that the good agreement between theory and simulations for the long pulses ( $150 \mathrm{fs})$ is partially due to another limitation of the theoretical model that disregards the effect of the transversal (radial) electron current from the periphery of the focal spot to its center. Such a flow of particles is able to

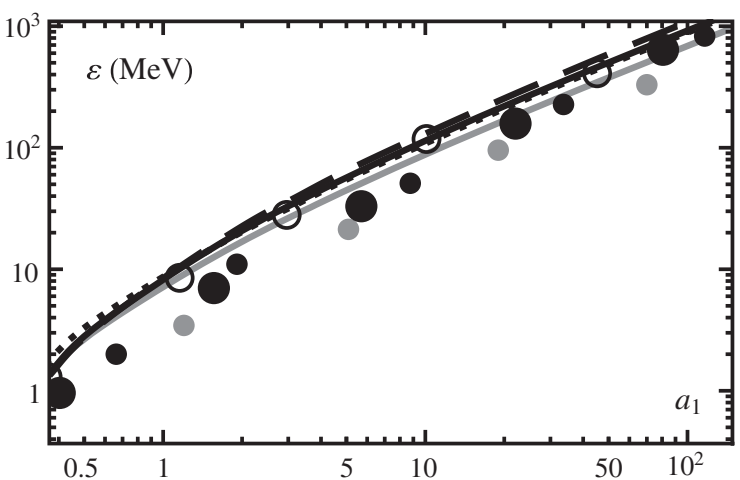

FIG. 6. Maximum proton energy vs absorbed laser field amplitude $a_{1}$. Circles represent the numerical simulations and lines are the theoretical results for the following laser parameters: $d=2 \mu \mathrm{m}, \tau=30$ fs (gray dots and solid gray line), $d=4 \mu \mathrm{m}, \quad \tau=30 \mathrm{fs}$ (small black dots and solid black line), $d=6 \mu \mathrm{m}, \tau=30$ fs (large black dots and dashed black line), $d=4 \mu \mathrm{m}, \tau=150$ fs (open circles and dotted black line).

provide more accelerated electrons on the rear side of the target for the long enough laser pulses. For $\tau>d / v_{\perp}$, this effect may be significant. The velocity $v_{\perp}$ changes from 0 to $c$ and the typical time of transversal electron motion, $\sim d / c$, will be in the order of a few tens of femtoseconds. Notwithstanding these approximations, Fig. 6 demonstrates that overall, our simple theory correctly describes dependence of the maximum proton energy on laser and plasma parameters.

\section{THE ROLE OF A LOW-DENSITY COATING}

We will examine the effect of the lower density coating of the optimal thickness targets on the efficiency of proton acceleration. We will introduce an aerogel-like layer on the front side of the target $[38,39]$ and compare this design with a standard solid density foil of optimum thickness for the same laser energy, focal spot size, and pulse duration. The aerogel layer with thickness of $3 \mu \mathrm{m}$ consists of carbon ions and electrons. The electron density is varied from $n_{c}$ to $5 n_{c}$. The maximum increase of the proton energy up to $30 \%$ (from $51 \mathrm{MeV}$ up to $65 \mathrm{MeV}$ ) was obtained for aerogel with density of $4 n_{c}$ (see Fig. 7). Qualitatively our finding corresponds to the result of Ref. [38] however the energy increase is not so dramatic because our target was already well optimized for the maximum efficiency of ion acceleration.

More importantly, we believe that the low-density coating may help in generating the high-energy protons even for the conditions when no noticeable energy increase of accelerated particles is expected. Mainly, the pure ultrathin solid density targets are very sensitive to laser pulse quality and require very high intensity contrast ratio on the ps time scale. However, a low-density coating can relax this requirement on the laser contrast ratio and make a laserbased high-energy ion source viable in practice. 

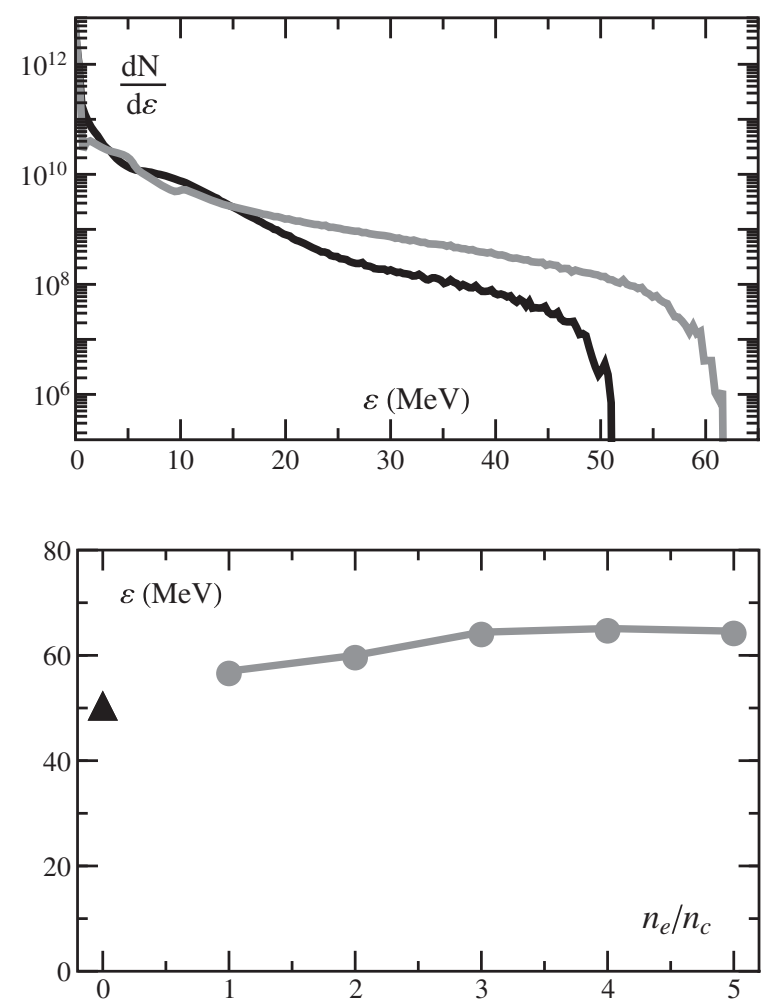

FIG. 7. Comparison of the spectra (upper panel) and maximum ion energies (bottom panel) for the solid density foil and twolayer target. The grey curve and dots correspond to the soliddensity foil with low-density coating and the black curve and the triangle correspond to pure overdense target.

\section{CONCLUSIONS}

We have studied proton acceleration from ultrathin targets with optimal thicknesses. For the first time, the absorption of laser light by semitransparent plasma has been described. This permits an understanding of laser intensity dependence of maximum proton energy, $\varepsilon \sim E_{l}^{0.7}$, for a wide range of laser energies (from 0.03 to $300 \mathrm{~J}$ ). A simple analytic theory has been proposed for the wide range of laser parameters. It agrees well with our simulation results. Both analytical and numerical predictions are quite optimistic about the proton energy gain even for laser intensities below the range required for the radiation pressure (light sail) regime. In general, experiments show that proton energy increases slower with laser intensity as compared to scaling predictions. We believe that the systematic experimental study with targets of optimum thicknesses and high contrast laser pulses should confirm the theoretical predictions of our paper.

\section{ACKNOWLEDGMENTS}

This work was supported by the Russian Foundation for Basic Research, the Russian Federation Presidential Program for State Support of Leading Scientific Schools, and the Natural Sciences and Engineering Research Council of Canada with the exception of Sec. IV which was supported by the Russian Science Foundation.

[1] V. Yu. Bychenkov, V. T. Tikhonchuk, and S. V. Tolokonnikov, J. Exp. Theor. Phys. 88, 1137 (1999).

[2] C. Labaune, C. Baccou, S. Depierreux, C. Goyon, G. Loisel, V. Yahia, and J. Rafelski, Nat. Commun. 4, 2506 (2013).

[3] P. K. Patel, A. J. Mackinnon, M. H. Key, T. E. Cowan, M. E. Foord, M. Allen, D. F. Price, H. Ruhl, P. T. Springer, and R. Stephens, Phys. Rev. Lett. 91, 125004 (2003).

[4] H.-S. Park et al., High Energy Density Phys. 8, 38 (2012).

[5] M. Borghesi, A. Schiavi, D. H. Campbell, M. G. Haines, O. Willi, A. J. MacKinnon, L. A. Gizzi, M. Galimberti, R. J. Clarke, and H. Ruhl, Plasma Phys. Controlled Fusion 43, A267 (2001).

[6] J. A. Cobble, R. P. Johnson, T. E. Cowan, N. Renard-Le Galloudec, and M. Allen, J. Appl. Phys. 92, 1775 (2002).

[7] L. Willingale, P. M. Nilson, M. C. Kaluza, A. E. Dangor, R. G. Evans, P. Fernandes, M. G. Haines, C. Kamperidis, R. J. Kingham, C. P. Ridgers, M. Sherlock, A. G. R. Thomas, M. S. Wei, Z. Najmudin, K. Krushelnick, S. Bandyopadhyay, M. Notley, S. Minardi, M. Tatarakis, and W. Rozmus, Phys. Plasmas 17, 043104 (2010).

[8] M. Roth, T. E. Cowan, M. H. Key, S. P. Hatchett, C. Brown, W. Fountain, J. Johnson, D. M. Pennington, R. A. Snavely, S. C. Wilks, K. Yasuike, H. Ruhl, F. Pegoraro, S. V. Bulanov, E. M. Campbell, M. D. Perry, and H. Powell, Phys. Rev. Lett. 86, 436 (2001).

[9] S. V. Bulanov and V. S. Khoroshkov, Plasma Phys. Rep. 28, 453 (2002).

[10] A. Henig, S. Steinke, M. Schnurer, T. Sokollik, R. Horlein, D. Kiefer, D. Jung, J. Schreiber, B. M. Hegelich, X. Q. Yan, J. Meyer-ter-Vehn, T. Tajima, P. V. Nickles, W. Sandner, and D. Habs, Phys. Rev. Lett. 103, 245003 (2009).

[11] F. Dollar, T. Matsuoka, G. M. Petrov, A. G. R. Thomas, S. S. Bulanov, V. Chvykov, J. Davis, G. Kalinchenko, C. McGuffey, L. Willingale, V. Yanovsky, A. Maksimchuk, and K. Krushelnick, Phys. Rev. Lett. 107, 065003 (2011).

[12] Y. Sentoku, V. Yu. Bychenkov, K. Flippo, A. Maksimchuk, K. Mima, G. Mourou, Z. M. Sheng, and D. Umstadter, Appl. Phys. B 74, 207 (2002).

[13] S. P. Hatchett et al., Phys. Plasmas 7, 2076 (2000).

[14] S. C. Wilks, A. B. Langdon, T. E. Cowan, M. Roth, M. Singh, S. Hatchett, M. H. Key, D. Pennington, A. MacKinnon, and R. A. Snavely, Phys. Plasmas 8, 542 (2001).

[15] K. Nishihara, H. Amitani, M. Murakami, S. V. Bulanov, and T.Z. Esirkepov, Nucl. Instrum. Methods Phys. Res., Sect. A 464, 98 (2001).

[16] V. F. Kovalev and V. Y. Bychenkov, J. Exp. Theor. Phys. 101, 212 (2005).

[17] S. S. Bulanov, A. Brantov, V. Yu. Bychenkov, V. Chvykov, G. Kalinchenko, T. Matsuoka, P. Rousseau, S. Reed, V. Yanovsky, D. W. Litzenberg, K. Krushelnick, and A. Maksimchuk, Phys. Rev. E 78, 026412 (2008). 
[18] L. Yin, B. J. Albright, B. M. Hegelich, K. J. Bowers, K. A. Flippo, T. J. T. Kwan, and J. C. Fernandez, Phys. Plasmas 14, 056706 (2007).

[19] T. Z. Esirkepov, M. Yamagiwa, and T. Tajima, Phys. Rev. Lett. 96, 105001 (2006).

[20] J. Fuchs, P. Antici, E. d'Humieres, E. Lefebvre, M. Borghesi, E. Brambrink, C. A. Cecchetti, M. Kaluza, V. Malka, M. Manclossi, S. Meyroneinc, P. Mora, J. Schreiber, T. Toncian, H. Pepin, and P. Audebert, Nat. Phys. 2, 48 (2006).

[21] D. V. Romanov, V. Yu. Bychenkov, W. Rozmus, C. E. Capjack, and R. Fedosejevs Phys. Rev. Lett. 93, 215004 (2004).

[22] P. Mora, Phys. Rev. Lett. 90, 185002 (2003).

[23] V. Yu. Bychenkov and V.F. Kovalev, Quantum Electron. 35, 1143 (2005).

[24] E. d'Humieres, A. V. Brantov, V. Yu. Bychenkov, and V. Tikhonchuk, Phys. Plasmas 20, 023103 (2013).

[25] K. I. Popov, V. Yu. Bychenkov, W. Rozmus, and R. D. Sydora, Phys. Plasmas 15, 013108 (2008).

[26] V. A. Vshivkov, N. M. Naumova, F. Pegoraro, and S. V. Bulanov, Phys. Plasmas 5, 2727 (1998).

[27] A. V. Brantov and V. Yu. Bychenkov, Contrib. Plasma Phys. 53, 731 (2013)
[28] A. Pukhov, Phys. Rev. Lett. 86, 3562 (2001).

[29] E. A. Govras and V. Yu. Bychenkov, JETP Lett. 98, 70 (2013).

[30] V. I. Arnold, Catastrophe Theory (Springer, Berlin, 1992).

[31] A. V. Gurevich and K. P. Zybin, Sov. Phys. JETP 67, 1 (1988).

[32] A. E. Kaplan, B. Y. Dubetsky, and P. L. Shkolnikov, Phys. Rev. Lett. 91, 143401 (2003).

[33] V. F. Kovalev, K. I. Popov, V. Yu. Bychenkov, and W. Rozmus, Phys. Plasmas 14, 053103 (2007).

[34] A. V. Gurevich and A. P. Meshcherkin, Sov. Phys. JETP 53, 937 (1981).

[35] J.E. Allen and M. Perego, Phys. Plasmas 21, 034504 (2014).

[36] K. I. Popov, V. Yu. Bychenkov, W. Rozmus, and L. Ramunno, Phys. Plasmas 17, 083110 (2010).

[37] V. F. Kovalev, V. Yu. Bychenkov, and V. T. Tikhonchuk, J. Exp. Theor. Phys. 95, 226 (2002).

[38] T. Nakamura, M. Tampo, R. Kodama, S. V. Bulanov, and M. Kando, Phys. Plasmas 17, 113107 (2010).

[39] M. Passoni, A. Zani, A. Sgattoni, D. Dellasega, A. Macchi, I. Prencipe, V. Floquet, P. Martin, T. V. Liseykina, and T. Ceccotti, Plasma Phys. Controlled Fusion 56, 045001 (2014). 